\title{
Reflexiones sobre el campo de las relaciones internacionales en Latinoamérica. Una mirada a partir de las publicaciones*
}

\section{RESUMEN}

Este trabajo presenta una reflexión sobre el desarrollo más reciente del campo de las relaciones internacionales en América Latina. Se apoya principalmente en un análisis bibliométrico de las principales revistas latinoamericanas que publican estudios afines, y en un análisis cualitativo de publicaciones

\author{
Adolfo A. Abadía** \\ Juan Pablo Milanese ${ }^{* * *}$ \\ Juan José Fernández Dusso****
}

* Este es un artículo de reflexión que se construye a partir de resultados del proyecto de investigación "Observatorio de Cooperación Internacional para el Desarrollo", aprobado y financiado por el Centro de Investigaciones CIEs de la Universidad Icesi.

** Politólogo. Estudiante de la Maestría en Estudios Sociales y Políticos de la Universidad Icesi, Cali (Colombia). Investigador y asistente editorial de la Oficina de Publicaciones de la Facultad de Derecho y Ciencias Sociales de la Universidad Icesi, Cali (Colombia). aaabadia@icesi.edu.co - http://orcid.org/0000-0002-9034-2156

*** Doctor en Ciencias Políticas y Sociales. Profesor del Departamento de Estudios Políticos, Universidad Icesi, Cali (Colombia).jmilanese@icesi.edu.co - http://orcid.org/0000-0003-0980-3435

**** Maestría en Ciencia Política. Profesor del Departamento de Estudios Políticos, Universidad Icesi, Cali (Colombia).jjfernandez@icesi.edu.co

Recibido: 6 de julio de 2015 / Modificado: 21 de septiembre de 2015 / Aceptado: 6 de abril de 2016

Para citar este artículo

Abadía, A. A., Milanese, J. P. y Fernández Dusso, J. J. (2016). Reflexiones sobre el campo de las relaciones internacionales en Latinoamérica. Una mirada a partir de las publicaciones. OAsıs, 23, 7-30.

DOI: http://dx.doi.org/10.18601/16577558.n23.02 
serie de reflexiones y sugerencias que buscan resaltar la importancia de un mayor debate teórico y, particularmente, metodológico para el fortalecimiento del estudio de la política internacional en -y desde- América Latina.

Palabras clave: estudios internacionales latinoamericanos, relaciones internacioanles, publicaciones, investigación, historia y desarrollo disciplinar, América Latina.

\section{Reflections on the study of international relations in Latin America. A review from the perspective of its publications}

\section{ABSTRACT}

This paper reflects on the most recent development of the international relations field in Latin America. It relies mainly on a bibliometric analysis of the main Latin American journals that publish related studies, and on a qualitative analysis of leading publications from some of the region's Political Science and International Relations departments. Without claiming to be an exhaustive study of the state of the field in the region, the paper includes new and useful information to build a reflection on the field's weaknesses, strengths and pending tasks. The work concludes with a series of reflections and suggestions that seek to highlight the importance of further theoretical and -particularly- methodological discussions to strengthen the study of international politics in -and from- Latin America.

Key words: Latin American international studies, international relations, publications, research, discipline's history and development, Latin America.

\section{INTRODUCCIÓN}

El estudio de las relaciones internacionales (RI) surge en el ámbito académico dentro de un marco temporal y contextual particular. Su punto de partida suele ubicarse en un periodo que arranca después de terminada la Primera Guerra Mundial ${ }^{1}$ (Neethling, 2004) y va hasta antes de la Guerra Fría (Barbér, 1987). El campo fue constituyéndose desde estudios sobre la historia diplomática, el derecho internacional y la diplomacia/geopolítica (Arenal, 1981, pp. 856-876; Tomassini, 1980, p. 547; Ortiz, 2011, p. 37). Su lugar de origen es el mundo anglosajón, con Estados Unidos como principal referente de este campo del conocimiento (Tickner, Cepeda y Bernal, 2013, p. 42-43).

Para Latinoamérica, la creación del Programa de Estudios Conjuntos sobre las Relaciones Internacionales de América Latina (RIAL), en 1977, representa un hito importante

\footnotetext{
$1 \quad$ Estudiosos de la historia de las relaciones internacionales como campo de investigación académica consideran la cátedra Woodrow Wilson, dictada en 1919 en la Universidad Aberystwyth de Gales por Alfred Eckhard Zimmern, un diplomático e historiador inglés, como el primer curso en el campo (Salomón, 2002, p. 5).
} 
en la historia del estudio de las relaciones internacionales en la región (Herz, 2008; Murillo Zamora, 2012; Tomassini, 1980). Su presencia puso en diálogo a un campo de estudio en proceso de consolidación como ciencia autónoma, en el marco de las ciencias sociales de la región, con Estados Unidos y Europa, y ayudó así a dar reconocimiento a la región como un área de importante madurez dentro del ámbito de los estudios sociales y de la política internacional en particular. La RIAL propició espacios tanto para el fortalecimiento metodológico y teórico, como para modelar líneas de investigación que procuraran analizar la participación de América Latina en el sistema político internacional (Tickner, 2002, pp. 120-121).

En lo que al estudio de la historia y el desarrollo del campo en la región respecta, tres momentos podrían identificarse. Un primer momento que coincide con la existencia de la RIAL, en el que se encuentran trabajos que propenden por mostrar la importancia y despertar el interés del estudio de las relaciones internacionales para América Latina (ver los trabajos de Lafer, 1978; Tomassini, 1980; Muńoz, 1980; Lagos Matus, 1980; Green, 1980). Un segundo momento que comprende las siguientes dos décadas tras la desaparación de la RIAL en 1991, en el que se destacan el trabajo doctoral de Arlene B. Tickner (2002) sobre los estudios internacionales en la región y el seminario "El estado de la disciplina de las Relaciones Internacionales en América" ${ }^{2}$ en 2008, y que buscan mostrar un balance del campo de las relaciones internacionales, principalmente desde una perspectiva de lo que se enseña y lo que se investiga. Y un tercer momento en el que se encuentran trabajos como el de Liliana Ramalho Fróio (2012) o Eduardo Devés-Valdés (2013), que indagan sobre la evolución temática y metodológia de los estudios de las relaciones internacionales, así como la encuesta Teaching, Research and Practice of International Relations (TRIP) del College of William and Mary, que lo hace sobre percepciones frente a diferentes asuntos académicos en el campo de las relaciones internacionales (en 2011, cabe decirse, se incluyen por primera vez cuatro países latinoamericanos en la encuesta: Argentina, Brasil, Colombia y México).

No obstante lo anterior, tras casi medio siglo de actividad del campo en América Latina, la información existente sobre los estudios internacionales es bastante dispersa, y las discusiones más álgidas sobre el campo han girado en torno a la preocupación por la construcción de teorías sobre relaciones internacionales autónomas y emancipadas; menos sobre las necesidades de fortalecer apuestas metodológicas específicas, o sobre la necesidad de empezar a reconocer y definir al campo como uno de estudios de política internacional, o incluso de estudios globales, más que como aquel del valioso pero limitado estudio de las relaciones internacionales (y es que claramente el campo, en la práctica

2 Seminario coordinado por Arlene B. Tickner y organizado por el Centro de Estudios y Programas Interamericanos (CEPI) del Departamento de Estudios Internacionales del Instituto Tecnológico Autónomo de México (ITAM). 
y desde hace un buen tiempo, ofrece una mayor variedad y riqueza en este aspecto $)^{3}$.

Este trabajo busca dar luz sobre estos asuntos mediante una aproximación al campo de las relaciones internacionales en Latinoamérica, a partir de un estudio bibliométrico de las publicaciones propias de ese ámbito -durante los últimos cuatro años- en las ocho principales revistas de la región, y de una revisión de la producción bibliográfica-de los últimos quince años- de los investigadores pertenecientes a varios de los departamentos académicos más destacados de la región. Sin el ánimo de constituirse en un estudio exhaustivo sobre la producción e investigación del campo en Latinoamérica, el objetivo de este artículo de reflexión es el de dar luz sobre matices que entendemos perdidos en buena parte del debate sobre la producción y el pensamiento latinoamericano al respecto y, particularmente, el de resaltar la necesidad de una mirada más modesta sobre lo que han sido los desarrollos teóricos desde estos estudios y lo que son sus tareas pendientes en aras de mayores aportes en este sentido (llamando con insistencia a atender más la necesidad de discusiones sobre el método para avanzar en dicha cuestión).

La estructura de este trabajo consta, introducción y conclusiones aparte, de tres secciones. La primera comprende un análisis bibliométrico de las ocho principales revistas de la región que publican estudios del cam- po de la política internacional. Se detallan allí aspectos de la internacionalización de las publicaciones a la luz de la inclusión en índices y rankings de alto impacto internacional, la diversidad de los temas, la proveniencia de los autores y los idiomas en que publican sus contenidos. La segunda sección traduce el análisis cuantitativo de la sección anterior, apoyado y complementado además por un recorrido a través de algunos de los principales departamentos y trabajos de investigadores de la región, en uno de carácter cualitativo en el que se destacan apuestas temáticas, metodológicas y teóricas de valor. Paso seguido, la tercera sección ofrece una mirada crítica sobre la producción académica del campo, principalmente en lo que a su aporte teórico y metodológico respecta, para finalmente esbozar algunas sugerencias que hacen hincapié en la necesidad de impulsar mayores discusiones metodológicas que procuren el avance y fortalecimiento de los estudios del campo.

\section{BREVE CARACTERIZACIÓN DE LAS RELACIONES INTERNACIONALES EN AMÉRICA LATINA A TRAVÉS DE UN ESTUDIO BIBLIOMÉTRICO}

Una de las formas más convencionales de rastrear la producción investigativa de los miembros de una comunidad académica es a través de la revisión de la publicación de

\footnotetext{
3 Probablemente, la estrategia de definir al campo como aquel de las Relaciones Internacionales, buscando diferenciase de la Ciencia Política e instalarse desde allí como una disciplina en propiedad, haya conspirado contra el mismo en este último sentido. (Sobre formas en que algunos autores dividen a la Ciencia Política en subcampos académicos, entre ellos las Relaciones Internacionales, ver ejemplos en Unesco, 1950; Goodin y Klingelmann, 1996; Cuéllar Argote, 2007; Vallès, 2008; Goodin, 2010; Fortou y Leyva Botero, 2013).
} 
sus artículos en revistas científicas. Si bien es cierto que estas no representan el único modo de difusión son, sin embargo, centrales como medio de comunicación. Su presencia en índices y rankings (caracterizados por un alto nivel de sistematización de la información y accesibilidad a los textos), permite además una revisión amplia, confiable y detallada de temas, problemas, apuestas teóricas y metodológicas, y formas de su presentación (en este caso hacemos especial referencia al uso de idiomas).

El presente apartado se propone identificar algunas de las principales tendencias que caracterizan a la investigación en el área de las relaciones internacionales en la región. Para lograrlo llevamos a cabo un análisis estadístico de carácter descriptivo basado en una serie de indicadores bibliométricos ${ }^{4}$. Para su realización privilegiamos aquellas revistas incluidas en el SCImago Journal Rank (sJR) (dentro de la categoría "Ciencia Política y Relaciones Internacionales" del área de Ciencias Sociales en Latinoamérica) $)^{5}$. Del total de once revistas que arrojó este filtro se identificaron tres mexicanas, dos colombianas, una de Chile, una de Brasil y otra más de Venezuela, para un total de ocho revistas académicas que, bajo las condiciones mencionadas, publican estudios sobre política internacional en la región (tabla 1).

TABLA 1. REVISTAS REGISTRADAS EN SJR EN RELACIONES INTERNACIONALES

\begin{tabular}{|l|l|l|l|}
\hline \multicolumn{2}{|c|}{ Revista } & \multicolumn{1}{|c|}{ Editorial } & \multicolumn{1}{c|}{ País } \\
\hline 1. & \multicolumn{1}{|c|}{$\begin{array}{c}\text { Migraciones } \\
\text { internacionales }\end{array}$} & $\begin{array}{l}\text { Colegio de la Frontera } \\
\text { Norte }\end{array}$ & México \\
\hline 2. & $\begin{array}{l}\text { Confines de relacio- } \\
\text { nes internacionales y } \\
\text { ciencia política }\end{array}$ & $\begin{array}{l}\text { Instituto Tecnológico y } \\
\text { de Estudios Superiores } \\
\text { de Monterrey }\end{array}$ & México \\
\hline 3. & Política y gobierno & $\begin{array}{l}\text { Centro de Investi- } \\
\text { gación y Docencia } \\
\text { Económicas }\end{array}$ & México \\
\hline 4. & $\begin{array}{l}\text { Colombia interna- } \\
\text { cional }\end{array}$ & $\begin{array}{l}\text { Universidad de los } \\
\text { Andes }\end{array}$ & Colombia \\
\hline 5. & Análisis político & $\begin{array}{l}\text { Universidad Nacional } \\
\text { de Colombia }\end{array}$ & Colombia \\
\hline 6. & $\begin{array}{l}\text { Revista de ciencia } \\
\text { política }\end{array}$ & $\begin{array}{l}\text { Pontificia Universidad } \\
\text { Católica de Chile }\end{array}$ & Chile \\
\hline 7. & $\begin{array}{l}\text { Revista brasileira } \\
\text { de política interna- } \\
\text { cional }\end{array}$ & $\begin{array}{l}\text { Instituto Brasileiro } \\
\text { de Relações Interna- } \\
\text { cionais }\end{array}$ & Brasil \\
\hline 8. & $\begin{array}{l}\text { Cuadernos del } \\
\text { Cendes }\end{array}$ & $\begin{array}{l}\text { Universidad Central } \\
\text { de Venezuela }\end{array}$ & Venezuela \\
\hline
\end{tabular}

Fuente: elaboración propia a partir de la información de SCImago Journal y Country Rank (sRJ)

\footnotetext{
4 Análisis similares fueron llevados a cabo, por ejemplo, por Tickner (2002), quien se enfoca en cinco revistas especializadas en temas de relaciones internacionales en América Latina: Colombia Internacional (Colombia), Contexto Internacional (Brasil), Estudios Internacionales (Chile), Foro Internacional (México) y Relaciones Internacionales (Costa Rica), en un marco temporal que arranca en 1960 y se extiende hasta 1998 (Ticker, 2002, p. 99). Para nuestra sorpresa, solo una de estas revistas está presente en este estudio pues es la única que ha logrado ser incluida en Scopus, uno de los principales referentes académicos a nivel mundial. Tres de ellas hacen parte de scielo, una librería electrónica de la producción científica de importante impacto a nivel iberoamericano.

5 scimago Journal Rank (sJR) es un portal que genera indicadores, principalmente, a partir de la información que recoge de la base de datos Scopus (Elsevier B.V.). Reconocemos que, como en cualquier selección de este tipo, existen argumentos arbitrarios para escoger las fuentes de la muestra. En nuestro caso, la razón se basa en el hecho de que dentro del ranking encontramos las principales revistas latinoamericanas tanto por la difusión como por el impacto de la publicación y de las citas realizadas.
} 
Cabe remarcar que, si bien las anteriores revistas publican estudios internacionales en un sentido amplio, cada una ha definido una serie de ejes temáticos principales que delimita sus contenidos. Así, por ejemplo, aunque la Revista brasileira de politica internacional y migraciones internacionales haya definido el estudio de los asuntos internacionales como eje temático principal, cada una ha delimitado su nicho de interés en la política exterior de Brasil y los estudios sobre migración internacional, respectivamente.

Por su parte, Confines de Relaciones Internacionales y Ciencia Politica y Colombia Internacional dejan en claro su interés tanto por estudios de ciencia política como de relaciones internacionales, constituyéndose como las publicaciones con mayor variedad temática. Mientras tanto, Política y gobierno, Análisis politico y la Revista de ciencia politica aseguran contribuir al conocimiento de estudios sobre temas y fenómenos políticos desde una perspectiva amplia de la ciencia política, incluyendo estudios relacionados con las relaciones internacionales. Finalmente, Cuadernos del Cendes se caracteriza por un foco temático más amplio, proponiendo un diálogo entre las diferentes corrientes del pensamiento de las ciencias sociales que se enfrentan a los problemas del desarrollo de América Latina y el mundo.
A partir de este marco, la primera dimensión que tendremos en cuenta es el nivel de internacionalización de las publicaciones como medio para valorar la producción académica regional, a la que evaluaremos de tres formas ${ }^{6}$. La primera de ellas consiste en identificar la presencia de las revistas en bases e índices de revistas académicas internacionales. En este sentido, la multiplicación de bases puede constituirse no solo en un referente de calidad sino también en un indicador de mayor presencia en los diferentes espacios de consulta académica, lo que aumentaría sus oportunidades de visualización (posteriormente, probablemente en citaciones) en los contenidos de las revistas académicas. La segunda implica el rastreo del nivel de internacionalización de las revistas a partir de la relación autores nacionales frente a autores internacionales. En este sentido, se esperaría que las revistas poseedoras de un mayor grado de internacionalización multipliquen sus posibilidades de atraer a un público más amplio en términos de lugar de origen, filiación institucional de los autores, etc. Finalmente, como tercera exploración, aparece la relación de la publicación de artículos en idiomas diferentes al nativo, principalmente, el inglés.

Si se observa el nivel de inclusión de las ocho revistas seleccionadas en los índices y rankings de revistas académicas (tabla 2), se

\footnotetext{
6 Destacamos Scopus (Elsevier) e IsI Web of Knowledge (Thomson Reuters), que tienen un alto impacto a nivel internacional y recogen publicaciones, principalmente, en inglés. Con impacto regional y producción principalmente en español y portugués están scielo (Brasil), RedALyC (México) y Dialnet (España). Todas las anteriores bases e índices de revistas manejan procesos de postulaciones y evaluación de las revistas para ser incluidas, por tanto, la principal diferencia entre ellas consiste en las barreras para acceder a los contenidos publicados. Mientras que las tres últimas son de acceso abierto (open access), la dos primeras no lo son.
} 
nota una presencia sistemática en portales especializados disciplinariamente bajo la sombrilla de Ciencia Política y Sociología como
International Political Science Abstracts, Political Science Complete, Worldwide Political Science Abstracts, así como Social Services

TABLA 2. ÍNDICES Y BASE DE DATOS DE LAS REVISTAS, 2011-2014

\begin{tabular}{|c|c|c|}
\hline Revista & ISSN & Índices y bases de revista \\
\hline $\begin{array}{l}\text { Migraciones interna- } \\
\text { cionales }\end{array}$ & $1665-8906$ & $\begin{array}{l}\text { Indexada en: Scopus, Academic Search Premier, Fuente Academica, Fuente Academica } \\
\text { Premier, International Political Science Abstracts, Political Science Complete, Social servi- } \\
\text { ces abstracts, Sociological abstracts, Worldwide Political Science Abstracts, Dialnet } \\
\text { Evaluada en: SRJ, DOAJ, Latindex, CIRC }\end{array}$ \\
\hline $\begin{array}{l}\text { confines de relaciones } \\
\text { internacionales y } \\
\text { ciencia política }\end{array}$ & $1870-3569$ & $\begin{array}{l}\text { Indexada en: Academic Search Premier, Fuente Académica, Fuente Académica Premier, } \\
\text { International Political Science Abstracts, Political Science Complete, Social Services Abs- } \\
\text { tracts, Sociological Abstracts, Worldwide Political Science Abstracts, Dialnet } \\
\text { Evaluada en: SRJ, DOAJ, Latindex, CIRC }\end{array}$ \\
\hline Política y gobierno & $1665-2037$ & $\begin{array}{l}\text { Indexada en: Social Science Citation Index, PAIS International, Social Services Abstracts, } \\
\text { Sociological Abstracts, Worldwide Political Science Abstracts } \\
\text { Evaluada en: SRJ, Latindex }\end{array}$ \\
\hline $\begin{array}{l}\text { Colombia interna- } \\
\text { cional }\end{array}$ & 0121-5612 & $\begin{array}{l}\text { Indexada en: Scopus, Academic Search Premier, Fuente Académica, Fuente Academica } \\
\text { Premier, International Bibliography of Social Sciences, International Political Science } \\
\text { Abstracts, PAIs International, Political Science Complete, Social Services Abstracts, Socio- } \\
\text { logical Abstracts, vLex, Worldwide Political Science Abstracts, Dialnet } \\
\text { Evaluada en: SRJ, DOAJ, Latindex, CIRC }\end{array}$ \\
\hline Análisis político & 0121-4705 & $\begin{array}{l}\text { Indexada en: Scopus, Periodicals Index Online, International Political Science Abstracts, } \\
\text { Political Science Complete, Social Services Abstracts, Sociological Abstracts, Worldwide } \\
\text { Political Science Abstracts } \\
\text { Evaluada en: SRJ, DOAJ, Latindex, CIRC }\end{array}$ \\
\hline $\begin{array}{l}\text { Revista de ciencia } \\
\text { política (Santiago de } \\
\text { Chile) }\end{array}$ & 0716-1417 & $\begin{array}{l}\text { Indexada en: Scopus, Social Science Citation Index, Academic Search Premier, Fuente } \\
\text { Académica, Fuente Academica Premier, International Bibliography of Social Sciences, } \\
\text { International Political Science Abstracts, Political Science Complete, Social Services Abs- } \\
\text { tracts, Sociological Abstracts, Worldwide Political Science Abstracts } \\
\text { Evaluada en: SRJ, DOAJ, Latindex, CIRC, CARHUS PLUS }\end{array}$ \\
\hline $\begin{array}{l}\text { Revista brasileira de } \\
\text { política internacional }\end{array}$ & $0034-7329$ & $\begin{array}{l}\text { Indexada en: Scopus, Social Science Citation Index, American History and Life, Academic } \\
\text { Search Premier, Fuente Académica, Fuente Académica Premier, International Bibliogra- } \\
\text { phy of Social Sciences, Periodicals Index Online, Historical Abstracts, International Politi- } \\
\text { cal Science Abstracts, Political Science Complete, Social services abstracts, Sociological } \\
\text { abstracts, Worldwide Political Science Abstracts } \\
\text { Evaluada en: SRJ, DOAJ, Latindex, CIRC, CARHUS PLUS }\end{array}$ \\
\hline $\begin{array}{l}\text { Cuadernos del } \\
\text { Cendes }\end{array}$ & $1012-2508$ & $\begin{array}{l}\text { Indexada en: Scopus, Periodicals Index Online, International Political Science Abstracts, } \\
\text { Political Science Complete, Social Services Abstracts, Sociological Abstracts, Worldwide } \\
\text { Political Science Abstracts, Dialnet } \\
\text { Evaluada en: SRJ, DOAJ, Latindex, CIRC }\end{array}$ \\
\hline
\end{tabular}

Fuente: elaboración propia a partir de la información de cada revista en el portal Matriz de Información para el Análisis de Revistas (MIAR) y sCImago Journal y Country Rank (sRJ) 
Abstracts y Sociological Abstracts, respectivamente. Posteriormente, la participación de las revistas en portales multidisciplinares como Scopus, Academic Search Premier, Fuente Academica, Fuente Academica Premier y Dialnet.

A simple vista, el panorama de las revistas seleccionadas es alentador en términos de la presencia en importantes portales de consulta por contenidos académicos. En este sentido, aumentan las probabilidades de que los estudios latinoamericanos en relaciones internacionales estén interactuando tanto con diferentes aproximaciones teóricas y metodológicas como con múltiples autores y temáticas. Esto genera un amplio abanico de posibilidades que permite a los investigadores acceder a un banco de contenidos que goza de un amplio reconocimiento internacional.

Para analizar los dos siguientes puntos -los lugares de origen de los autores y los idiomas de publicación-se revisaron un total de 157 artículos entre el 2011 y 2014, entre ellos 102 fueron de autor único, 44 escritos en coautoría, dentro de los cuales en 9 hay 3 investigadores y en 2 se encuentran 4 investigadores para un gran total de 225 autores. Como puede observarse, el $35 \%$ de los artículos se hicieron en colaboración y aproximadamente la mitad de estas coautorías se da entre colegas afiliados a una misma institución. Luego, y en una similar proporción, las publicaciones son producto de procesos de colaboración nacional o internacional.
Con el objetivo de evidenciar algunos patrones, las revistas se agruparon por países (tabla 3). Cruzados los resultados con variables como la filiación institucional de los autores, pueden apreciarse dos tendencias claras: por un lado, en aproximadamente el $50 \%$ de los casos coinciden la nacionalidad del autor y de la revista, observándose picos en los casos de Brasil y Colombia, en donde el 67 y el $55 \%$ de los artículos pertenecen a autores del mismo origen. Por el otro, son solamente un puñado los que concentran una notable mayoría de las publicaciones. En este sentido, los investigadores de Brasil, Colombia, Argentina y España (29,3, 15,6, 12,4 y $10,7 \%$, respectivamente) producen alrededor del $70 \%$ del total de los artículos de los journals mencionados, evidenciando una notable concentración. Finalmente, es interesante destacar que la Revista brasileira de politica internacional es en la que se refleja una mayor diversidad en términos del origen de los autores, mientras que los artículos de aquellos provenientes de España se han distribuido más parejamente que cualquier otro.

Para una mirada sobre las temáticas de las publicaciones se revisaron las palabras clave de los distintos artículos, observándose un total de $715^{[7]}$. Se logró agrupar a 282 en 30 denominadores temáticos para un total de 418 (tabla 4). Las agrupaciones responden al establecimiento de relaciones según un orden geoespacial (Brasil, China, América Latina, Colombia, Estados Unidos, Suramé-

\footnotetext{
7 Con un promedio de cinco por artículo y una oscilación entre dos y doce, número mínimo y máximo en algunos casos, respectivamente.
} 
TABLA 3. REVISTAS Y AUTORES SEGÚN PAÍS DE ORIGEN, 2011-2014

\begin{tabular}{|c|c|c|c|c|c|c|c|}
\hline \multirow{3}{*}{\begin{tabular}{|l} 
País autor(es) \\
Argentina
\end{tabular}} & \multicolumn{5}{|c|}{ País de las revistas } & & \\
\hline & Brasil & Chile & Colombia & México & Venezuela & \multicolumn{2}{|c|}{ Total general (\%) } \\
\hline & 4 & & 6 & 14 & 4 & 28 & $(12,4)$ \\
\hline Austria & & & 1 & & & 1 & $(0,4)$ \\
\hline Bélgica & & & 2 & & & 2 & $(0,9)$ \\
\hline Brasil & 60 & 2 & 3 & 1 & & 66 & $(29,3)$ \\
\hline Canadá & & & 3 & & & 3 & $(1,3)$ \\
\hline Chile & 2 & 3 & & 4 & & 9 & $(4,0)$ \\
\hline Colombia & & & 32 & 3 & & 35 & $(15,6)$ \\
\hline EE.UU. & 2 & & 4 & 5 & & 11 & $(4,9)$ \\
\hline Escocia & & & 1 & & & 1 & $(0,4)$ \\
\hline España & 3 & 2 & 2 & 12 & 5 & 24 & $(10,7)$ \\
\hline Francia & 1 & & & 2 & & 3 & $(1,3)$ \\
\hline Holanda & & & 1 & & & 1 & $(0,4)$ \\
\hline Inglaterra & 1 & & 1 & 2 & & 4 & $(1,8)$ \\
\hline México & 2 & & 1 & 11 & & 14 & $(6,2)$ \\
\hline Portugal & 7 & & & 2 & & 9 & $(4,0)$ \\
\hline Suiza & 2 & & & & & 2 & $(0,9)$ \\
\hline Taiwán & 2 & & & & & 2 & $(0,9)$ \\
\hline Turquía & 1 & & & & & 1 & $(0,4)$ \\
\hline Uruguay & 2 & & & & & 2 & $(0,9)$ \\
\hline Venezuela & & & 1 & & 5 & 6 & $(2,7)$ \\
\hline Vietnam & 1 & & & & & 1 & $(0,4)$ \\
\hline \multirow[t]{2}{*}{ Total general (\%) } & 90 & 7 & 58 & 56 & 14 & 225 & \\
\hline & $(40)$ & (3) & (26) & (25) & (6) & & (100) \\
\hline
\end{tabular}

Fuente: elaboración propia a partir de los contenidos de scielo y Redalyc de cada revista.

rica, Unión Europea, Asia, Venezuela, África y Argentina), conceptos afines (Migración, Política Exterior, Teoría de las RI, Integración Regional, Seguridad, Cooperación Internacio- nal, Mercosur, Democracia, Estado, Guerra, TLC Bilaterales, Ri y Poder) o la constitución de conceptos clave por sí mismos (Sur global, organizaciones internacionales, globalización, 
derechos humanos, política exterior brasileña, transnacional). Los 388 denominadores restantes poseen una frecuencia menor de tres y no logran constituirse como denominadores clave.
La tabla 5 deja en evidencia los principales focos temáticos de los artículos publicados de acuerdo con las revistas y su origen. A partir de esta premisa podemos observar que aquellos presentados en la Revista brasileira de política

TABLA 4. CLASIFICACIONES GENERALES DE LOS TEMAS EN LAS REVISTAS, 2011-2014

\begin{tabular}{|c|c|c|c|}
\hline & Palabras clave & Denominad. utilizado & Frec.* \\
\hline 1 & $\begin{array}{l}\text { Migración laboral, Migración transgeneracional, Migrantes argentinos, Políticas migrato- } \\
\text { rias, Inmigración, Derechos humanos de migrantes, Proyectos migratorios, Inmigrantes } \\
\text { bolivianos, Red migratoria, Emigración, Vulnerabilidad de migrantes }\end{array}$ & Migración & 19 \\
\hline 2 & $\begin{array}{l}\text { Brazilian diplomacy, Brazil's international insertion, história das relações internacionais } \\
\text { do Brasil, Dilma Rousseff Administration, Governo Lula, Governo Fernando Henrique } \\
\text { Cardoso }\end{array}$ & Brasil & 16 \\
\hline 3 & Política exterior china, China's rise, South China Sea & China & 16 \\
\hline 4 & Relaciones exteriores, Política exterior & Política exterior & 16 \\
\hline 5 & $\begin{array}{l}\text { Liberalismo, Neoliberalism, Realismo, Constructivismo, Soft power, Anarquía, Hard } \\
\text { power, Great powers behavior, Constructivismo realista, Structural realism, }\end{array}$ & Teoría de las RI & 16 \\
\hline 6 & Latin America foreign affairs, América Latina, Latin América & América Latina & 13 \\
\hline 7 & Integración, Integración Andina, Regionalism, Unasur, Alba & Integración Regional & 13 \\
\hline 8 & $\begin{array}{l}\text { Seguridad internacional, Políticas de seguridad, Common foreign and security policy, } \\
\text { National Defense, National Defense Strategies, National security, national strategies, } \\
\text { Regional security, Regionalismo estratégico, Armed Forces, Defense }\end{array}$ & Seguridad & 13 \\
\hline 9 & Derechos humanos & DD.HH. & 12 \\
\hline 10 & $\begin{array}{l}\text { Cooperação Técnica Internacional, Cooperación interregional, Cooperación Militar, Coo- } \\
\text { peration, Regional Cooperation, Asistencia militar, Multilateralismo, Multilateral trade } \\
\text { negotiations }\end{array}$ & $\begin{array}{l}\text { Cooperación interna- } \\
\text { cional }\end{array}$ & 11 \\
\hline 11 & $\begin{array}{l}\text { Conflicto armado en Colombia, Gobierno Uribe, Plan Colombia, Violencia colombiana, } \\
\text { Administración Santos }\end{array}$ & Colombia & 10 \\
\hline 12 & uS Foreign Policy, US, Estados Unidos, EE.UU. & Estados Unidos & 10 \\
\hline 13 & Parlamento del Mercosur, Mercosur & Mercosur & 10 \\
\hline 14 & Política exterior brasileira & $\begin{array}{l}\text { Política exterior } \\
\text { Brasileira }\end{array}$ & 10 \\
\hline 15 & Chile, Bolivia, Paraguay & Sur América & 9 \\
\hline 16 & EU decision, Ciudadanía europea, Parlamento Europeo, EU & Unión Europea & 9 \\
\hline 17 & Southeast Asia, Taiwán, Vietnam, Hong Kong, Guerra de Corea & Asia & 7 \\
\hline
\end{tabular}




\begin{tabular}{|c|c|c|c|}
\hline & Palabras clave & Denominad. utilizado & Frec.* \\
\hline 18 & $\begin{array}{l}\text { Definiciones de democracia, Democracia participativa, Democracia representativa, De- } \\
\text { mocratización, Calidad democrática }\end{array}$ & Democracia & 7 \\
\hline 19 & Nación, Sistema de Estado, Soberanía & Estado & 7 \\
\hline 20 & Globalización & Globalización & 7 \\
\hline 21 & World War II, Guerra Fría, Posguerra fría, Economía de guerra & Guerra & 7 \\
\hline 22 & $\begin{array}{l}\text { Tratados comerciales en Perú y Chile, Acuerdos de libre comercio, Tratados de Libre } \\
\text { Comercio de EE.UU., Relaciones entre Colombia y Estados Unidos, Relaciones argentino- } \\
\text { brasileñas }\end{array}$ & TLC Bilaterales & 6 \\
\hline 23 & Inflación en Venezuela, Venezuela & Venezuela & 6 \\
\hline 24 & África & África & 5 \\
\hline 25 & Buenos Aires, Argentina & Argentina & 5 \\
\hline 26 & Ámbito internacional, Sistema internacional, Relaciones internacionales & RI & 5 \\
\hline 27 & Transnacional & Transnacional & 5 \\
\hline 28 & Organizaciones internacionales & $\begin{array}{l}\text { Organizaciones inter- } \\
\text { nacionales }\end{array}$ & 4 \\
\hline 29 & Relaciones de poder, Poder estatal, Poder judicial, Poderes emergentes, Biopoder & Poder & 4 \\
\hline 30 & South Cooperation & Sur Global & 4 \\
\hline
\end{tabular}

Fuente: elaboración propia a partir de los contenidos de scielo y Redalyc de cada revista.

* Once denominadores con frecuencia de 3, quince con frecuencia de 2 y trescientos setenta con 1 frecuencia. Para un total de 418 diferentes denominadores clave.

TABLA 5. PRINCIPALES TEMÁTICAS Y REVISTAS SEGÚN SU PAÍS DE ORIGEN, 2011-2014

\begin{tabular}{|c|c|c|c|c|c|c|c|}
\hline \multirow{3}{*}{$\begin{array}{l}\text { Denominador temático* } \\
\text { 1. Migración }\end{array}$} & \multicolumn{5}{|c|}{ País de las revistas } & & \\
\hline & Brasil & Chile & Colombia & México & Venezuela & \multicolumn{2}{|c|}{ Total general (\%) } \\
\hline & & & 1 & 18 & & 19 & $(6,7)$ \\
\hline 2. Política exterior & 5 & 2 & 5 & 3 & 1 & 16 & $(5,7)$ \\
\hline 3. Teoría de las RI & 6 & 1 & 8 & 1 & & 16 & $(5,7)$ \\
\hline 4. Brasil & 14 & & & 2 & & 16 & $(5,7)$ \\
\hline 5. China & 12 & 1 & 3 & & & 16 & $(5,7)$ \\
\hline 6. Seguridad & 9 & 1 & 2 & 1 & & 13 & $(4,6)$ \\
\hline 7. América Latina & 6 & & 3 & 4 & & 13 & $(4,6)$ \\
\hline 8. Integración regional & 3 & & 5 & 4 & 1 & 13 & $(4,6)$ \\
\hline
\end{tabular}




\begin{tabular}{|c|c|c|c|c|c|c|c|}
\hline \multirow{3}{*}{$\begin{array}{l}\text { Denominador temático* } \\
\text { 9. Derechos humanos }\end{array}$} & \multicolumn{5}{|c|}{ País de las revistas } & & \\
\hline & Brasil & Chile & Colombia & México & Venezuela & \multicolumn{2}{|c|}{ Total general (\%) } \\
\hline & 2 & & 8 & 2 & & 12 & $(4,3)$ \\
\hline 10. Cooperación internacional & 4 & & 5 & & 2 & 11 & $(3,9)$ \\
\hline 11. Estados Unidos & 3 & 1 & 4 & 2 & & 10 & $(3,5)$ \\
\hline 12. Mercosur & 3 & & 3 & 3 & 1 & 10 & $(3,5)$ \\
\hline 13. Colombia & & & 7 & 2 & 1 & 10 & $(3,5)$ \\
\hline 14. Política exterior brasileira & 9 & & 1 & & & 10 & $(3,5)$ \\
\hline 15. Suramérica & 5 & & 1 & 2 & 1 & 9 & $(3,2)$ \\
\hline 16. Unión Europea & 5 & 1 & 3 & & & 9 & $(3,2)$ \\
\hline 17. Estado & 1 & & 4 & 1 & 1 & 7 & $(2,5)$ \\
\hline 18. Asia & 4 & 1 & 2 & & & 7 & $(2,5)$ \\
\hline 19. Guerra & 2 & 1 & 3 & 1 & & 7 & $(2,5)$ \\
\hline 20. Democracia & 1 & & 2 & 4 & & 7 & $(2,5)$ \\
\hline 21. Globalización & & 1 & 1 & 4 & 1 & 7 & $(2,5)$ \\
\hline 22. TLC & & & 5 & 1 & & 6 & $(2,1)$ \\
\hline 23. Venezuela & & & 1 & 1 & 4 & 6 & $(2,1)$ \\
\hline 24. Argentina & 1 & & & 4 & & 5 & $(1,8)$ \\
\hline 25. Transnacional & & & & 5 & & 5 & $(1,8)$ \\
\hline 26. RI & 1 & 1 & & 3 & & 5 & $(1,8)$ \\
\hline 27. África & 3 & & 1 & 1 & & 5 & $(1,8)$ \\
\hline 28. Sur global & 1 & & 1 & 2 & & 4 & $(1,4)$ \\
\hline 29. Poder & & & 3 & 1 & & 4 & $(1,4)$ \\
\hline 30. Org. internacionales & 2 & & 2 & & & 4 & $(1,4)$ \\
\hline \multirow[t]{2}{*}{ Total general (\%) } & 102 & 11 & 84 & 72 & 13 & 271 & \\
\hline & (36) & (4) & (30) & (26) & (5) & & (100) \\
\hline
\end{tabular}

Fuente: elaboración propia a partir de los contenidos de scielo y Redalyc de cada revista.

* Sobre la clasificación de las palabras clave según denominador temático ver en anexos tabla 2.

internacional se concentran en cuestiones como la política exterior de los distintos gobiernos brasileños o historias de la inserción de este país en el sistema internacional. También se destaca el interés por el estudio de los acercamientos y el interés del Gobierno chino en la 
región. Es interesante destacar que existe una concentración temática por número dada la naturaleza monográfica de cada uno de ellos. A diferencia de este caso, en el de Colombia internacional no se presentan picos temáticos de semejante intensidad y, no obstante poseer volúmenes monográficos, podemos observar una mayor estabilidad de los temas de un número destacándose asuntos como la teoría de las relaciones internacionales, los derechos humanos y fenómenos ampliamente estudiados en el país como conflicto armado.

Por último, en lo referido a las revistas mexicanas el foco investigativo está puesto sobre las políticas y los procesos del fenómeno migratorio. Como puede observarse, y es de esperar, en cada uno de los casos existe un interés manifiesto por cuestiones que afectan de manera directa la agenda de política exterior de cada uno de los países. Esto no solamente es evidente en cada una de las publicaciones sino que se refuerza cuando, por ejemplo, se realiza una revisión de otro tipo de indicadores como los principales debates existentes en los congresos académicos.

En este sentido, si revisamos los programas de los últimos tres congresos de la Asociación Latinoamericana de Ciencia Política (concentrándonos específicamente en los paneles de RI) podemos observar claramente cómo el número de estos y el de ponencias varía sensiblemente de acuerdo con las principales problemáticas e intereses del país anfitrión (Argentina, 2010; Ecuador, 2012; Colombia, 2013) ${ }^{8}$.

\section{GRÁFICO 1. AÑO E IDIOMA DE PUBLICACIÓN} DE LOS ARTÍCULOS, 2011-2014

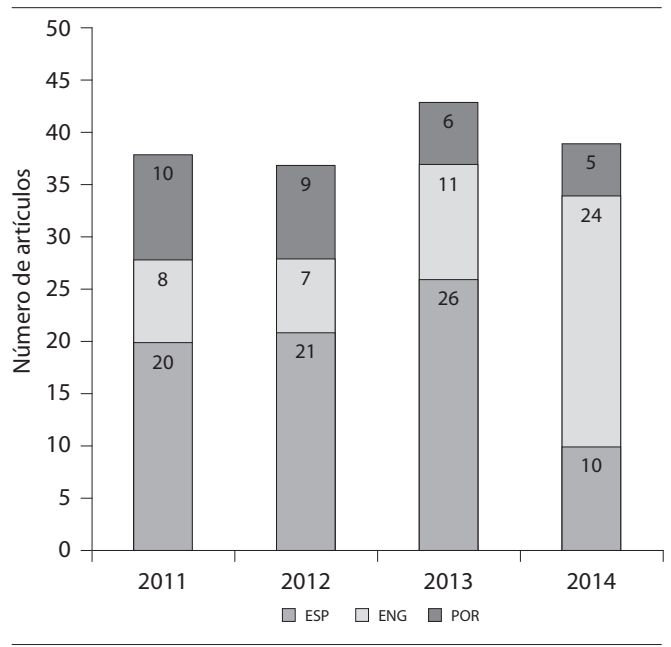

Fuente: elaboración propia a partir de los contenidos de scieLo y Redalyc de cada revista.

El siguiente paso consiste en hacer una lectura más precisa con respecto al grado de internacionalización de las revistas académicas analizadas, a partir de la variable idioma de los artículos publicados (gráfico 1). Dentro del marco temporal se observa, como es de esperar, el predominio del español frente al inglés y el portugués. Con una constante y llamativa reducción del número de publicaciones en portugués. Al analizar la distribución por año, puede observase además un cambio abrupto en 2014, para el que notamos un significativo crecimiento de los artículos en inglés. Y, si bien no contamos con información suficiente para asegurar que esto representa un momento de quiebre, no deja de ser un fenómeno interesante que invita a ser atendido.

\footnotetext{
8 Mayor información sobre los programas de los Congreso ALACIP en http://alacip.org/?page_id=3940
} 
En concordancia con lo dicho hasta el momento, pueden identificarse ciertas particularidades que dan luz a la hora de analizar el nivel de internacionalización de las publicaciones (y por tanto de la producción) en términos de la relación revistas y enfoques temáticos de los artículos y autor(es).

En primer lugar, se observa una inclinación a publicar en revistas del país de origen. Principalmente sobre temas de interés localnacional y en idioma español. En este sentido, tiende a hacerse evidente una producción académica focalizada en problemáticas definidas, más claramente visible en las revistas mexicanas y en la brasileña, cuyos intereses temáticos parecen ser mucho más delimitados que los de las publicaciones revisadas en Chile, Colombia y Venezuela.

En esto juega un rol evidente el desarrollo temático de cada publicación que, como es natural, no es ajeno a la agenda existente en cada país. Así, no sorprende que en México exista una revisa sobre migraciones y que cubra más del $95 \%$ de los artículos identificados con ese denominador temático dentro de la muestra trabajada.

En segundo lugar, como se mencionó, el español presenta un predominio evidente. Sin embargo, es interesante tener en cuenta el visible crecimiento de las publicaciones en inglés registrado entre el 2011 y 2014. Se registra un aumento muy significativo en el número de artículos publicados en ese idioma, aunque es importante reconocer que la mayor parte de estos se concentra en la Revista brasileira de politica internacional y en Colombia internacional de 2014 (ver anexo tabla 1).

\section{DEL NÚMERO A LA SUSTANCIA: REVISIÓN CUALITATIVA DE LAS APUESTAS TEÓRICAS DE ALGUNOS DEPARTAMENTOS ACADÉMICOS EN LA REGIÓN}

Una de las dimensiones del análisis propuesto por este trabajo es la del aporte de académicos latinoamericanos del campo a la teoría de las relaciones internacionales. La revisión bibliométrica del aparte anterior da luces para entender cómo temas y problemas específicos han sido más o menos abordados recientemente desde el campo según revistas latinoamericanas y lugar de origen de los investigadores. También, al indicar lugares, idiomas e incluso colaboraciones investigativas a los que se apuesta para la publicación, da luces sobre la pretensión de alcance e impacto que los autores puedan tener para dar visibilidad a sus resultados, alimentar debates, construir redes o consolidar agendas.

La revisión bibliométrica, sin embargo, dice todavía poco sobre el aporte del pensamiento latinoamericano a la teoría de las relaciones internacionales. El presente aparte complementa el análisis ofreciendo una revisión de apuestas teóricas y metodológicas específicas que permitan entender el nivel de desarrollo del campo de las relaciones internacionales en América Latina, y el potencial que pueda estar teniendo para afectar el debate teórico o la agenda académica internacional.

Para el presente apartado el análisis es construido a partir de la identificación de algunos de los principales departamentos de Ciencia Política y Relaciones Internacionales de la región. Utilizamos como punto de partida la selección realizada por Altman (2011) 
en su preocupación por descifrar el aporte de diferentes departamentos latinoamericanos al campo de la Ciencia Política en general, y le sumamos algunos departamentos más que entendemos, a la luz de su aporte al campo de las relaciones internacionales (asunto atendido tangencialmente por Altman) o a su crecimiento más reciente, vale la pena incorporar'. En este sentido, también se tuvieron en cuenta otras consideraciones relevantes como la incorporación de algunos países que no estaban incluidos en la muestra y, sobre todo, la ampliación de aquellos departamentos existentes en Colombia dando muestra de la diversidad regional en el nivel subnacional. Así, los departamentos revisados-admitiendo que puede haber omisiones importantes- pertenecen a las instituciones que se muestran en la tabla 6.

Desde allí recorremos de forma general las áreas de trabajo de los investigadores pertenecientes a cada departamento dedicados al

TABLA 6. UNIVERSIDADES CON DEPARTAMENTOS Y FACULTADES DE RELACIONES INTERNACIONALES

\begin{tabular}{|l|l|l|}
\hline \multicolumn{1}{|c|}{ Argentina } & \multicolumn{1}{|c|}{ Chile } & \multicolumn{1}{|c|}{ México } \\
\hline Universidad de Buenos Aires & Pontificia Universidad Católica de Chile & CIDE \\
\hline Universidad de San Andrés & Universidad de Chile & El Colegio de México, A.C. \\
\hline Universidad del Salvador & Universidad Diego Portales & $\begin{array}{l}\text { Instituto Tecnológico Autónomo } \\
\text { de México }\end{array}$ \\
\hline Universidad Nacional de Rosario & & \multicolumn{1}{|c|}{ Colombia } \\
\hline Universidad Torcuato Di Tella & & Perú \\
\hline Universidad Nacional de San Martín & Pontificia Universidad Javeriana & Universidad Católica del Perú \\
\hline & Universidad de los Andes & Uruguay \\
\hline Brasil & Universidad del Norte & Eniversidad de la República \\
\hline Pontificia Universidad Católica do Río de Janeiro & Universidad del Rosario & \\
\hline Universidad Cándido Mendes & Universidad Eafit & Flacso \\
\hline Universidad de São Paulo & Universidad Externado de Colombia \\
\hline Universidad Estadual de Campinas & Universidad Icesi & \\
\hline Universidad Federal de Minas de Gerais & Universidad Nacional & \\
\hline Universidad Federal de Río Grande do Sul & & \\
\hline
\end{tabular}

Fuente: elaboración propia.

9 La relevancia de sus papeles se hace evidente a lo largo de nuestro ejercicio de revisión bibliométrica. También frente al reconocimiento de investigadores, adscritos a estos, con publicaciones en revistas o libros no exclusivamente regionales y de reconocimiento académico internacional. 
estudio de la política internacional, y los aportes específicos que a través de sus publicaciones ${ }^{10}$ hayan procurado para la construcción y discusión teórica o metodológica concerniente al campo de las relaciones internacionales ${ }^{11}$.

En principio, debe decirse que los resultados generales de la primera parte del análisis son consecuentes -y complementarios-con lo hallado en los apartes del análisis bibliométrico, en cuanto se encuentra una gran y variada cantidad de temas que develan numerosos esfuerzos de investigación sobre asuntos de particular importancia para la región (migraciones, seguridad, integración regional, China como nuevo actor fundamental dentro del comercio -y la economía política-internacional, derechos humanos y cooperación); se encuentra que la mayor parte de las publicaciones es escrita en espańol (seguida por una porción importante en portugués); y que dentro del mayoritario número de publicaciones que se hacen dentro de la región, las revistas del país de residencia del investigador predominan como oportunidad para la divulgación, dando unas primeras señales de lo que sería una todavía débil red académica de estudios internacionales y de correspondientes debates regionales y comparados sobre la experiencia y las apuestas investigativas en la región ${ }^{12}$.

También debe decirse que los resultados generales son consecuentes $-\mathrm{y}$ complemen- tarios- con lo indicado por Devés-Valdés (2013, pp. 49-51) en lo que respecta al uso predominante de perspectivas provenientes de los "países centrales"; a la baja relevancia de problemas regionales ${ }^{13}$ para la academia anglosajona (evidenciada en sus publicaciones y agenda de investigación); a la necesidad o conveniencia de la constitución de una comunidad epistémica hasta ahora débil (a pesar de esfuerzos colaborativos que se evidencian en asociaciones, congresos e incluso algunas publicaciones compartidas -como se muestra en la revisión bibliométrica-); y a la posibilidad de "capitalizar lo que se elabora en otras periferias" en aras de la construcción de nuevas y originales aproximaciones teóricas y metodológicas al estudio de los fenómenos de la política internacional (temas que serán abordados con mayor profundidad en el próximo apartado).

No obstante lo dicho anteriormente, se reconocen ejercicios de discusión teórica claramente valiosos, que aportan al debate y a la comprensión de asuntos específicos (y, en varios casos, de relevancia también para la literatura anglosajona), como son, por solo mencionar algunos, los trabajos de Roberto Bouzas sobre liberalización económica, acuerdos comerciales e iniciativas de integración que, desde el estudio de experiencias latinoamericanas, plantean además -y críticamente-

\footnotetext{
10 En este aparte el periodo en revisión es mayor: aportes en publicaciones durante los últimos quince años.

11 La existencia de una apuesta teórica o metodológica original y explícita, así como el tipo y lugar de la publicación (revisadas a partir de sus cvs), fueron las consideraciones en juego para iniciar un análisis cualitativo de ciertos autores o trabajo particulares.

12 En este sentido, el reconocimiento de la literatura sobre el asunto da mayor respaldo a esta primera insinuación.

13 Que, como se señalara, cubren la mayor parte de los ejercicios de investigación.
} 
implicaciones políticas de dichos procesos para la región. O los de Federico Merke sobre teoría de las relaciones internacionales y del derecho internacional; de Andrea Betti sobre el derecho internacional; los de Marta Fernández Moreno sobre relaciones internacionales, misiones internacionales y seguridad; R. B. J. Walker sobre el futuro de las democracias; los de Ernesto Vivares sobre financiación internacional para el desarrollo sobre y periferias y semiperiferias; de Cintia Quiliconi sobre regionalismo, economía internacional y desarrollo sustentable; o de investigadores como Élodie Brun, Paulo Fagundes Vizentini o Analucía Danilevicz Pereira sobre relaciones Sur-Sur.

La selección de estos autores, aunque arbitraria como cualquier ejercicio de este tipo, no es fortuita sino hecha para demostrar que:

a) La diversidad de asuntos, problemas y actores propios de la política internacional, como lo seńalan Devés-Valdés (2013, pp. 58-59), pero también otros tantos autores más, desborda las justificadas preocupaciones sobre -la formalidad de- la política exterior de los Estados de la región, propias de autores centrales dentro del debate sobre la teoría de las relaciones internacionales en América Latina (como Raúl Bernal-Meza, Arlene Tickner, Fernanda Barasuol, Mónica Herz, Amado Cervo, Roberto Russell, Nicolás Onuf o Edmundo Heredia, para mencionar algunos de los más destacados).

b) Aun siendo escaso el acercamiento a investigaciones, apuestas teóricas y metodológicas de otras latitudes (periféricas), algunos pocos esfuerzos en este sentido están siendo llevados a cabo en la región (aún no representativos para el contexto, cabe decirse).

c) No obstante la preocupante escasez de discusiones sobre metodología en el campo, existen ejercicios que procuran una sistematización y explicación de casos con un mayor rigor metodológico.

d) Aun siendo predominante el uso de perspectivas provenientes de los "países del centro", la diversidad de preocupaciones y argumentos frente a problemas específicos permiten entrever potenciales debates internos valiosos para alimentar la generación de nuevo conocimiento y reflexión.

e) No obstante ser paradigmas y modelos metafóricos (el patrón boomerang, el juego de dos niveles, la teoría del rol, etc.), más que complejos modelos teóricos, las herramientas preponderantes en el campo para la presentación de explicaciones sobre asuntos de política internacional, su uso aunado a ejercicios de, por ejemplo, política comparada, pueden resultar en aportes para una construcción de teorías de mediano alcance o para la rigurosa validación, complementación o falsación de teorías preexistentes dentro o fuera de la región (tarea colectiva, creemos, aún pendiente).

f) Existe una visible asimetría (naturalmente con indiscutibles excepciones) entre el nivel del campo alcanzado por los distintos países de la región (encabezados por Brasil, México y Argentina) y el resto. Algo similar ocurre entre las distintas 
vertientes del campo: algunas como los análisis de seguridad, de derecho internacional o de asuntos comerciales parecen haber alcanzado un mayor rigor analítico en general.

\section{REFLEXIONES Y SUGERENCIAS PARA EL DESARROLLO DEL CAMPO EN AMÉRICA LATINA}

Lo revisado hasta ahora permite reconocer la existencia de una importante producción en el campo de las relaciones internacionales a nivel regional, que evidencia un significativo grado de profesionalización y especialización. Sin embargo, esta producción sigue estando acompańada de una insatisfacción estructural de parte de la academia que reclama la falta de desarrollo de unas relaciones internacionales más latinoamericanas.

Este escenario es precisamente señalado por Devés-Valdés (2013), quien realiza un minucioso y lúcido trabajo de reflexión "acerca de la posibilidad y/o necesidad de una teorización latinoamericana, así como una discusión sobre la necesidad y los eventuales aportes de esta” (p. 48).

El autor resalta que no es nuevo el intento por incentivar una producción vernácula -debate instalado por autores como Luciano Tomassini, Rubén Perina o Juan Carlos Puig- que reemplace la existencia de teorías “importadas” que se aplican sin ser necesariamente las más adecuadas para el análisis regional (Tickner, 2003; 2011). Esa necesidad de producción teórica de carácter autóctono comenzó progresivamente a ganar peso en la agenda del campo, acompañada por intentos más esporádicos de apelar a aquella proveniente de otros espacios periféricos que permitan revertir la tendencia de baja densidad de intercambios con estos últimos y de la circulación unidireccional de conocimiento desde el norte hacia el sur (Barasoul, 2010).

No obstante, en relación con los argumentos previamente esgrimidos existe una ineludible pregunta que todavía hay que repetir: ¿para qué el desarrollo de estas teorías? Es decir, ¿cuál es el objetivo por el que una empresa semejante cobra sentido?

El ejercicio de revisión de la producción del campo en América Latina nos ha permitido encontrar la persistente existencia de llamados a crear una teoría latinoamericana que parta de posiciones emancipadoras desde un punto de vista político. En este caso, en lugar de la producción de herramientas que permitan una mejor lectura y sistematización de la realidad (asunto mayoritariamente relegado a un segundo plano), lo que se encuentra es la poderosa presencia de demandas de reivindicación de carácter ideológico. Y legítimas como son para el debate sobre el lugar de los países de la región dentro de las dinámicas de la política internacional, débiles lo son para la construcción y justificación de teorías robustas y complejas, y problemáticas además si tenemos en cuenta el intrínseco riesgo de lysenkismo que algunas de estas posturas tienden a producir.

Débiles son para la construcción y justificación de teorías robustas y complejas al relegar la necesidad de debates metodológicos que procuren aproximaciones más rigurosas al estudio de sus problemas. Débiles también lo son al tratar de justificar paradigmas o me- 
táforas (útiles, valiosos, quepa decirse) como teorizaciones dignas de la mayor elegancia argumentativa y complejidad. Riesgosas lo son, en muchos casos, por la orgullosa defensa de lo anterior ${ }^{14}$.

Creemos, entonces, que la respuesta a la pregunta sobre el desarrollo de nuevas teorías debe pasar, sobre todo y principalmente, por la intención de enriquecer los análisis con escenarios mejor contextualizados y herramientas más precisas y adecuadas.

Aunque podamos notar una tendencia creciente en los debates metodológicos dentro del campo, esta es aún modesta y, entretanto, persiste una notable pobreza desde este punto de vista en muchas investigaciones propias del campo de las relaciones internacionales latinoamericanas. De hecho, y sin manifestar una preferencia específica por estudios orientados a casos o a variables ${ }^{15}$, debemos decir que abundan aún estudios basados en información no sistematizada o contrastada brindada por “informantes", prensa ${ }^{16} \mathrm{o}$, peor aún, inspiradas intuiciones. En este sentido, en repetidas oportunidades puede llegarse a dudar de si asistimos a rigurosos trabajos de investigación o a apreciaciones informadas de elegantes "analistas de coctel" 17.

Dicho lo anterior, lo que sugerimos aquí es la procura de una empresa colectiva de producción de teorías de alcance medio (Merton, 1980), a través de análisis comparados que permitan diagnósticos $-y$ recomendaciones de política- más precisos y complejos para las realidades locales y de esta u otra región. Hacemos referencia a un escenario caracterizado por preguntas e "hipótesis que no pretendan lograr una unificación (teórica) ideal, sino integrar campos limitados pero significativos de investigación” (Cataño, 2006, p. 383). Un escenario de este tipo, caracterizado por conceptos con un nivel de abstracción medio (Sartori, 2004), contribuiría tanto a evitar la utilización de categorías inadecuadas para el contexto regional como las utilizadas exclusivamente para el análisis de casos específicos $y$, por tanto, incapaces de contribuir a un proceso de producción de teoría o de original y eficaz incidencia sobre políticas - públicas, comerciales, diplomáticas- particulares. Una

14 Importa recordar experiencias reivindicadoras de un pensamiento latinoamericano más sofisticado y particular, que ponen necesarios matices a esta afirmación. Tal es el caso de la Escuela de Brasilia (Bernal-Meza, 2005), útil además como ejemplo para soportar nuestras proposiciones generales de las próximas páginas.

15 Superando el innecesario debate sobre métodos cuantitativos frente a cualitativos, consideramos que no existe una metodología intrínsecamente más adecuada, sino que la selección de esta (o estas) deberá depender de la pregunta de investigación.

16 Hacemos referencia, en este caso, no a análisis que buscan entender la lectura de la prensa sobre la política internacional, o que pretendan sistematizaciones juiciosas de información brindadas durante significativos periodos por la misma, sino a casos en que se justifican tesis o argumentaciones a partir de información brindada -sin mayores soportes explícitos o solicitados- por periodistas o columnistas.

17 De manera recurrente nos encontramos con trabajos que no se diferencian significativamente de versiones en "formato académico" del trabajo que pueda realizar un cronista. Artículos de opinión con referencias bibliográficas y exploración -a menudo poco profunda y no sistematizada- de información escasamente contrastada. 
empresa de este tipo rendiría sólidos frutos para soportar, además, la defensa de las ya mencionadas y legítimas demandas emancipadoras, garantizándole probablemente más adeptos al liberarla de sus debilidades propias e inherentes riesgos.

Consideramos importante, sin embargo, recalcar sobre el punto anterior que, en el afán de desarrollar esta producción, debemos ser extremadamente cuidadosos para evitar las miradas de excepcionalidad sobre la situación de América Latina. Mucho más sensato es en este sentido pensar en la particularidad de la región (y en sus similitudes con otras): la idea de una excepcionalidad podría llevarnos a una situación de autismo inconducente. Por tanto, no deberíamos repudiar con prejuicio los conceptos caracterizados por un alto nivel de abstracción (en buena parte producidos en el "centro académico"), por considerarlos inercialmente vagos, políticamente sospechosos, o culture-bound, produciendo así "cacerías de brujas" (Sartori, 2004). De hecho, en este sentido consideramos que pueden ser útiles los procesos de hibridación (Tickner, 2011) que permitan, simultáneamente, entender mejor la problemáticas regionales pero también dialogar con la producción proveniente de otras partes del mundo (posibilidad, esta última, que decrece a medida que disminuye el nivel de abstracción).

Pero esta hibridación no debería pensarse exclusivamente desde un punto de vista geográfico. También es importante hacerlo desde un punto de vista disciplinario. Podría ser extraordinariamente enriquecedor apelar a herramientas provenientes de otras disciplinas o subcampos del análisis político como la sociología histórica, la -ya sugerida- política comparada en sus distintas vertientes, las políticas públicas o nuevos enfoques de la teoría política contemporánea. Lamentablemente, esto pareciera chocar a veces con expectativas exageradas de reconocimiento disciplinar, atentando contra la posibilidad de construcción de teorías políticas y sociales más complejas.

Autores como los citados tras la revisión de departamentos de la región dan cuenta de la posibilidad de lograr estos fines. Preocupación por la sofisticación del método, ejercicios de hibridación, acercamiento a otras experiencias, apuestas de construcción y fortalecimiento de teorías complejas y robustas, pueden ir dando forma paulatinamente a una comunidad epistémica más autónoma y sólida (aunque heterogénea por los requerimientos de especialización a los que la realidad llama), generando efectos significativos para la comprensión de -y la acción sobre- fenómenos específicos de países y regiones.

\section{CONCLUSIONES}

La revisión de las más recientes publicaciones del campo en las ocho revistas latinoamericanas más importantes para el mismo, nos habla de una amplia gama de temas y problemas que demandan distintos tipos de abordaje y esfuerzos de teorización. Buena parte de estos temas o problemas responden a fenómenos sociales y políticos propios de -países de- la región. Algunos otros, referentes por ejemplo a asuntos de derecho internacional, globalización y democracia, pueden permitirse un juego de mayor alcance en términos de la 
discusión y afectación de teorías más generales (comúnmente provenientes de la academia "del centro").

La amplia gama de temas y problemas, además, viene acompańada del reconocimiento de un significativo número de investigadores propios del ámbito, que la revisión de departamentos corrobora. Crecimiento del campo, diversificación de los asuntos estudiados y mayores grados de especialización consecuente durante los últimos años son las primeras conclusiones que pueden empezar a sugerirse desde aquí. De alguna manera, y según lo identificado por esta revisión, lo que indica Devés-Valdés (2013, p. 58) citando a Lessa (2005) sobre los cambios en la Escuela de Brasilia reflejarían transformaciones reconocibles dentro del campo general en América Latina: "Lessa (2005, p. 16) señala que la reflexión brasileña sobre RRII ganó en sofisticación y multidisciplinariedad, entre el inicio de los noventa y mediados de la primera década del xxi”. Pero con salvedades todavía necesarias:

La revisión de los departamentos seleccionados da cuenta de disparidades en términos de la sofisticación mencionada atrás, por países y por temas, tanto en términos teóricos como metodológicos.

Pero la revisión también recoge la persistencia de debates epistemológicos visibles dentro del campo, con autores relevantes provenientes de distintos departamentos y países de la región. Y esto, junto a lo anterior, resulta sugestivo al momento de afrontar posibilidades de construcción y transformación del pensamiento -y aporte- latinoamericano en lo referente al campo. Nuestra intención consiste, sin embargo, en insistir sobre la necesidad de una mayor discusión metodológica para el campo de los estudios de la política internacional en América Latina -en toda su diversidad-. Las desiguales falencias del ámbito en términos de construcción de nuevas teorías (robustas, complejas), y de explicaciones rigurosas de los casos de estudio, llaman con apremio a esto.

\section{REFERENCIAS}

Abadía, A. A. (2015). Del Liberalismo al Neo-realismo. Un debate en torno al Realismo clásico. TELOS: Revista Interdisciplinaria en Ciencias Sociales, $17(3)$

Altman, D. (2006). From Fukuoka to Santiago: Institutionalization of Political Science in Latin America. PS: Political Science and Politics, 39 (1), 196-203.

Altman, D. (2011). Where is Knowledge Generated? On the Productivity and Impact of Political Science Departments in Latin America. European Political Science, 11 (1), 71-87.

Álvarez Valdés, R. (2008). Chile y el estudio de las Relaciones Internacionales: ¿un caso paradójico? Seminario "El estado de la disciplina de las Relaciones Internacionales en América". México D.F.: Centro de Estudios y Programas Interamericanos (CEPI), Departamento de Estudios Internacionales, Instituto Tecnológico Autónomo de México (ITAM).

Arenal, C. D. (1981). La génesis de las relaciones internacionales como disciplina científica. Revista de Estudios Internacionales, 2 (4), 849-892.

Barasoul, F. (2010). Teorização (in)dependente: as teorias de relaçôes internacionais e a formulação da politica externa brasileira. Trabalho de conclusão 
em Relações Internacionais, Porto Alegre, R. S., Universidade Federal do Rio Grande do Sul.

Barbé, E. (1987). El papel del realismo en las relaciones internacionales (La teoría de la política internacional de Hans J. Morgenthau). Revista de Estudios Politicos (Nueva Época) (57), 149-176.

Bernal-Meza, R. (2005). América Latina en el mundo: el pensamiento latinoamericano y la teoría de las Relaciones Internacionales. Buenos Aires: Grupo Editor Latinoamericano.

Bulcourf, P., Gutiérrez Márquez, E. y Cardozo, N. (2015). Historia y desarrollo de la ciencia política en América Latina: reflexiones sobre la constitución del campo de estudios. Revista de Ciencia Política, 35 (1), 179-199.

Cardona Restrepo, P., Patiño Aristizabal, L. G., Silva Moyano, M. y Vieco Maya, L. E. (2013). Estado actual de la producción científica de los grupo de investigación en Ciencia Política y de las revistas en el país, 2002-2011. En Leyva Botero, S. (ed.). La Ciencia Politica en Colombia: ¿una disciplina en institucionalización?, (pp. 219-267). Medellín: Colciencias, ACCPOL, Centro de Análisis Político - Universidad EAFIT.

Cataño, G. (2006). Robert K. Merton. Espacio Abierto, 15 (1-2), 369-389.

Cuéllar Argote, J. (2007). Un diagnóstico a la enseñanza de la Ciencia Política en Colombia. Civilizar, $7(13)$.

Devés-Valdés, E. (2013). Cómo pensar los asuntos internacionales-mundiales a partir del pensamiento latinoamericano: análisis de la teorización. História Unisinos, 17 (1), 48-60.

Fortou, J. A. y Leyva Botero, S. (2013). Un análisis bibliométrico de microcurrículos de Ciencia Política en Colombia. En Leyva Botero, S. (ed.). La Ciencia Politica en Colombia: ¿una disciplina en institucionalización? (pp. 189-215). Medellín,
Colombia: Colciencias, ACCPOL, Centro de Análisis Político - Universidad EAFIT.

Goodin, R. E. y Klingemann, H. D. (1996). A New Handbook of Political Science. Oxford: Oxford University Press.

Goodin, R. E. (2010). The State of the Discipline, the Discipline of the State. En Robert E. Goodin (ed.). The Oxford Handbook of Political Science. Oxford: Oxford University Press.

Green, R. (1980). La importancia del estudio de las relaciones internacionales de los países latinoamericanos. Estudios Internacionales, 13 (52), 527-544.

Greenstein, F. y Polsby, N. (1975). Handbook of Political Science (vol. 9). Reading; Addison-Wesley.

Herz, M. (2008). The Study of International Relations in Latin America. Seminario "El estado de la disciplina de las Relaciones Internacionales en América". México D.F.: Centro de Estudios y Programas Interamericanos (CEPI), Departamento de Estudios Internacionales, Instituto Tecnológico Autónomo de México (ITAM).

Jaramillo, G. (2008). El estado de la disciplina de las Relaciones Internacionales en el Ecuador. Seminario "El estado de la disciplina de las Relaciones Internacionales en América”. México D.F.: Centro de Estudios y Programas Interamericanos (CEPI), Departamento de Estudios Internacionales, Instituto Tecnológico Autónomo de México (ITAM).

Lafer, C. (1978). El estudio de las relaciones intemacionales: necesidades y perspectivas. Estudios Internacionales, 11 (43), 47-56.

Lagos Matus, G. (1980). Tendencias y perspectivas del estudio de las relaciones internacionales: tareas para América Latina. Estudios Internacionales, 13 (50), 236-251.

Leyva, S. y Ramírez, M. F. (2015). La ciencia política en Colombia: una disciplina en contínua expanción. Revista de Ciencia Politica, 35 (1), 71-94. 
Lessa, A. C. (2005). Instituiçóes, atores e dinâmicas do ensino e da pesquisa em Relaçôes Internacionais no Brasil: o diálogo entre a história, a ciência política e os novos paradigmas de interpretação (dos anos 90 aos nossos dias). Revista Brasileira de Politica Internacional, 48 (2), 169-184.

Merke, F. (2008). El campo de las Relaciones Internacionales en la Argentina. Seminario "El estado de la disciplina de las Relaciones Internacionales en América”. México D.F.: Centro de Estudios y Programas Interamericanos (CEPI), Departamento de Estudios Internacionales, Instituto Tecnológico Autónomo de México (ITAM).

Merton, Robert K. (1980). Sobre las teorías sociológicas de alcance intermedio. En Teoría y estructura sociales (pp. 56-91). México, D.F.: Fondo de Cultura Económica.

Merton, R. K. (1968). On Sociological Theories of the Middle Range. En Social Theory and Social Structure (pp. 39-72). New York: Free Press.

MIAR (2015). Resultados. En Matriz de Información para el Análisis de Revistas (MIAR). Recuperado de http://www.miar.ube.edu/

Muñoz, H. (1980). Los estudios internacionales en América Latina: problemas fundamentales. Estudios Internacionales, 13 (51), 328-344.

Murillo Zamora, C. (2012). Estudiar Relaciones Internacionales en América Latina. Recuperado de http:/www.esglobal.org/estudiar-relacionesinternacionales-en-america-latina/

Neethling, T. (2004). The development of normative theory in International Relations: Some practical implications for norm-based and value-based scholarly inquiry. Koers, 69 (1), 1-25.

Ortiz, E. (2011). El estudio de las relaciones internacionales. Chile: Fondo de Cultura Económica.

Ramalho Fróio, L. (2012). O estado da arte das pesquisas em Relaçóes Internacionais na América
Latina. $8^{\circ}$ Encontro da Associaçāo Brasileira de Ciência Politica. Gramado: Associação Brasileira de Ciência Política (АВСР).

Robinson, J. (2008). Moctezuma’s Revenge: The State of IR in Mexico. Seminario "El estado de la disciplina de las Relaciones Internacionales en América". México D.F.: Centro de Estudios y Programas Interamericanos (CEPI), Departamento de Estudios Internacionales, Instituto Tecnológico Autónomo de México (ITAM).

Salomón, M. (2002). La teoría de las relaciones internacionales en los albores del siglo xxi: diálogo, disidencia, aproximaciones. Revista electrónica de estudios internacionales - REEI (4).

Sartori, G. (2004). La política. Lógica y método en las ciencias sociales. México: Fondo de Cultura Económica.

sCImago (2007). SJR - scimago Journal y Country Rank. Recuperado de http://www.scimagojr.com

Suárez Salazar, L. (2008). El desarrollo de los estudios sobre las Relaciones Internacionales en Cuba: apuntes para una actualización. Seminario "El estado de la disciplina de las Relaciones Internacionales en América”. México D.F.: Centro de Estudios y Programas Interamericanos (CEPI), Departamento de Estudios Internacionales, Instituto Tecnológico Autónomo de México (ITAM).

Tickner, A. B. (2002). Los estudios internacionales en América Latina ¿Subordinación intelectual o pensamiento emancipatorio? Bogotá, Colombia: Universidad de los Andes, CESO, Deptartamento de Ciencia Política: Alfaomega Colombiana.

Tickner, A. B. (2003). Pedagogy in International Studies: Hearing Latin American Voices in International Relations Studies. International Studies Perspectives, 4, 235-250.

Tickner, A. B. (2011). Relaciones de conocimiento centro-periferia: hegemonía, contribuciones 
locales e hibridización. Recuperado de www. cedep.ifch.ufrgs.br/

Tickner A. B. (2013). By Way of Conclusion: Forget IR? En Arlene, B., Tickner y David L. Blaney (eds.). Claiming the International (pp. 214-232). London: Routledge.

Tickner A. B., Cepeda, C. y Bernal, J. L. (2013). ¿Quiénes son los internacionalistas en Latinoamérica? Foreign Affairs Latinoamerica, 13 (2), 42-49.
Tomassini, L. (1980). Los estudios internacionales en América Latina: algunas contribuciones. Estudios Internacionales, 13 (52), 545-552.

Unesco (1950). Contemporary Political Science: A Survey of Methods, Research and Teaching. París, Francia: Organización de las Naciones Unidas para la Educación, la Ciencia y la Cultura (Unesco), Lieja G. Thone.

Vallès, J. (2008). Ciencia política: una introducción. Barcelona: Ariel.

ANEXOS

TABLA 1. NÚMERO DE PUBLICACIONES SEGÚN AÑO E IDIOMA, 2011-2014

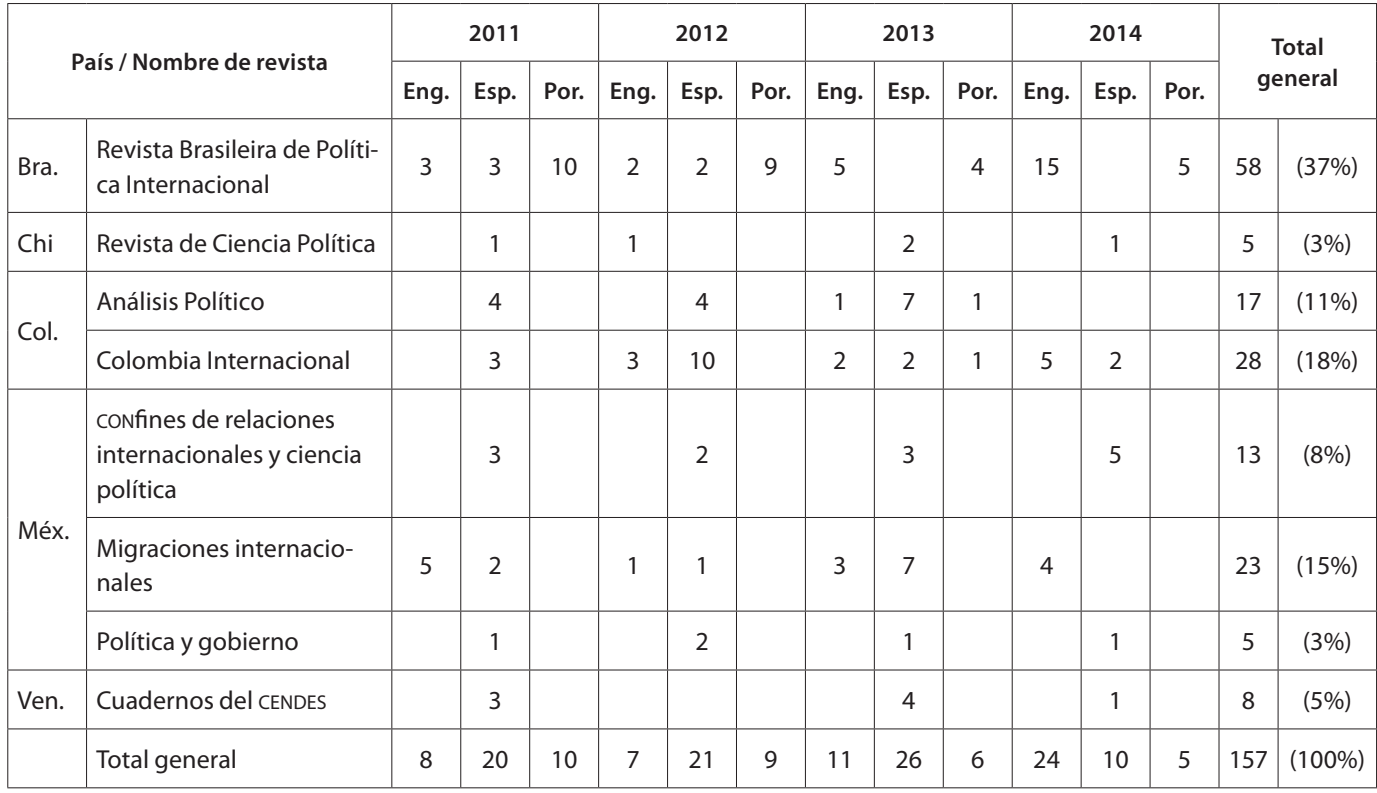

Fuente: elaboración propia a partir de los contenido de Scielo y Redalyc de cada revista. 